\title{
Efecto de la densidad de siembra sobre el peso corporal y sobrevivencia de larvas de bagre rayado (Pseudoplatystoma sp) en un sistema cerrado de circulación
}

\author{
The effect of rearing density on body weight and \\ survival of larval stripped catfish (Pseudoplatystoma \\ $s p)$ into a closed circulating system
}

\author{
Efeito da densidade de cultivo sobre o peso corporal e a sobrevivência \\ de larvas do Sorubim (Pseudoplatystoma sp) num sistema fechado de \\ circulação
}

\author{
Germán Castañeda $A^{1 *}$, Pablo Felipe Cruz-Ochoa ${ }^{2 *}$, Gilberto Moraes ${ }^{3 * * *}$, \\ Martha Yossa $P^{4 *}$, Pablo E. Cruz-Casallas $5^{*}$
}

1 Zoot, MSc;

$2 \mathrm{MV}$;

3 Cienc Biol., MSc, PhD;

4 Zoot, MSc, PhD; ${ }^{5} \mathrm{MVZ}, \mathrm{MSc}, \mathrm{PhD}$

* Universidad de los Llanos, Instituto de Acuicultura, Facultad de Ciencias Agropecuarias y Recursos Naturales, Km 12 vía Puerto López, Villavicencio, Meta, Colombia

** Departamento de Patología da Faculdade de Medicina Veterinária da Universidade de São Paulo, São Paulo, SP, Brasil

*** Centro de Ciências Biológicas e da Saúde, Departamento de Genética e Evolução, Universidade Federal de São Carlos, Rodovia Washington Luís, km 235, SP-310, São Carlos, São Paulo, Brasil.

E-mail: pecruzcasallas@unillanos.edu.co

\section{Resumen}

Con el propósito de evaluar los efectos de la densidad de siembra sobre el peso corporal y la sobrevivencia de larvas de bagre rayado (Pseudoplatystoma sp), fue diseñado y construido un sistema cerrado de recirculación, el cual permitió mantener estables las variables físicas y químicas del agua durante todo el período experimental. Para este propósito, larvas vitelinas fueron sembradas en el sistema a tres densidades, así: 10 (T1), 50 (T2) y 100 (T3) larvas por litro y monitoreadas hasta los 20 días post-eclosión. Fueron evaluadas cuatro réplicas por tratamiento, mantenidas bajo fotoperiodo 0:24 (luz:oscuridad) y alimentadas hasta aparente saciedad con nauplios de Artemia salina recién eclosionados, los cuales fueron suministrados repartidos en cinco raciones por día: a las 07:00, 09:00, 14:00, 18:00 y 22:00 horas. Las características de calidad de agua observadas durante el ensayo fueron: oxígeno disuelto $5.7 \pm 0.5 \mathrm{mg} / \mathrm{L} ;$ temperatura $26 \pm 0.8^{\circ} \mathrm{C} ; \mathrm{pH} 7.6 \pm 0.2 ;$ dureza $38.2 \pm$ $5.2 \mathrm{mgCaCO} 3 / \mathrm{L}$; alcalinidad 26.5 $\pm 5.2 \mathrm{mg} \mathrm{CaCO} 3 / \mathrm{L} ; \mathrm{NH} 3<0.02 \mathrm{mg} / \mathrm{L}$; salinidad $0.4 \pm 0.1 \mathrm{~g} / \mathrm{L}$; conductividad $841.1 \pm 38.1$ $\mu \mathrm{S} / \mathrm{cm}$. Al final del ensayo, el peso corporal varió inversamente a la densidad de siembra: $103.0 \pm 15.6 ; 76.1 \pm 19.8$ y 66.7 $\pm 9.3 \mathrm{mg}$, respectivamente, pero no se observaron diferencias significativas entre las dos densidades más altas $\mathrm{P}>0.05)$. La sobrevivencia fue mayor en la densidad de 10 larvas/L, siendo diferente estadísticamente entre tratamientos $(86.5 \pm 1.9 \%$; $54.7 \pm 2.2 \%$ y $46.9 \pm 3.1 \%$ para 10,50 y 100 larvas/L, respectivamente, $\mathrm{P}<0.01)$. En conclusión, la densidad de siembra afecta inversamente el peso corporal y la sobrevivencia de las larvas en esta especie. 
Palabras clave: peso corporal, densidad, larvas de peces, Pseudoplatystoma sp, supervivencia (Fuente: AIMS).

\begin{abstract}
In order to evaluate the effects of larval density rearing on body weight and survival of larvae of striped catfish (Pseudoplatystoma sp), was designed and built a closed recirculation system, which allowed maintaining stable physical and chemical variables of water throughout the experimental period. For this purpose, vitelline larvae were rearing in three densities: 10 (T1), 50 (T2) and 100 (T3) larvae per liter and monitored until 20 days post-hatch. They were evaluated four replicates per treatment, kept under photoperiod 0:24 (light: dark) and fed to apparent satiety with Artemia saline hatchlings, which were supplied divided into five portions a day: at 07:00, 09: 00, 14:00, 18:00 and 22:00 hours. The water quality characteristics observed during the test were: dissolved oxygen $5.7 \pm 0.5 \mathrm{mg} / \mathrm{L}$; temperature $26 \pm 0.8^{\circ} \mathrm{C} ; \mathrm{pH} 7.6 \pm 0.2 ;$ hardness $38.2 \pm$ $5.2 \mathrm{mgCaCO} 3 / \mathrm{L}$; alkalinity $26.5 \pm 5.2 \mathrm{mg} \mathrm{CaCO} 3 / \mathrm{L}$; NH3<0.02 mg/L; salinity $0.4 \pm 0.1 \mathrm{~g} / \mathrm{L}$; conductivity $841.1 \pm 38.1 \mu \mathrm{S} /$ $\mathrm{cm}$. At the end of the test, body weight varied inversely with rearing density: $103.0 \pm 15.6 ; 76.1 \pm 19.8$ and $66.7 \pm 9.3 \mathrm{mg}$, respectively, but no significant difference between the two highest densities $\mathrm{P}>0.05$ ) were observed. Survival was higher in the density of 10 larvae/L, being statistically different between treatments $(86.5 \pm 1.9 \%, 54.7 \pm 2.2 \%$ and $46.9 \pm 3.1 \%$ for 10,50 and 100 larvae/L, respectively, $\mathrm{P}<0.01)$. In conclusion, larval density inversely rearing affects body weight and survival of the larvae in this species.
\end{abstract}

Keywords: body weight, density, fish larvae, Pseudoplatystoma sp, survival (Source: AIMS).

\begin{abstract}
Resumo
A fim de avaliar os efeitos da densidade de estocagem sobre o peso corporal e a sobrevivência de larvas de Sorubim (Pseudoplatystoma sp), foi desenhado e construído um sistema de recirculação fechado, o que permitiu manter as variáveis físicas e químicas da água estáveis durante todo o período experimental. Para o efeito, larvas vitelinas foram semeadas no sistema em três densidades asim: 10 (T1), 50 (T2) e 100 (T3) larvas por litro e monitoradas até 20 dias pós-eclosão. Foram avaliados quatro repetições por tratamento, mantidas sob fotoperíodo 0:24 (luz: escuro) e alimentadas até saciedade aparente com nauplios de Artemia salina, que foram fornecidos divididos em cinco porções por dia: às 07:00, 09: 00, 14:00, 18:00 e 22:00 horas. As características de qualidade de água observada durante o ensaio foram: oxigénio dissolvido de

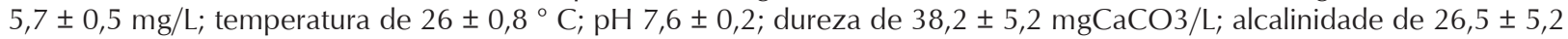
$\mathrm{mg} \mathrm{CaCO} 3$ / I; NH3 <0,02 mg/L; salinidade de 0,4 \pm 0,1 g/L; condutividade 841,1 $\pm 38.1 \mu \mathrm{S} / \mathrm{cm}$. No final do teste, o peso corporal variou inversamente com a densidade de estocagem: 103,0 $\pm 15,6 ; 76,1 \pm 19,8$ e 66,7 \pm 9,3 mg, respectivamente, mas não se observaram diferenças significativas entre as duas densidades mais altas $P>0,05)$. A sobrevivência foi maior na densidade de 10 larvas/L, o que foi estatisticamente diferente entre os tratamentos $(86,5 \pm 1,9 \%, 54,7 \pm 2,2 \%$ e 46,9 \pm $3,1 \%$ para 10, 50 e 100 larvas/L, respectivamente, $P<0,01)$. Em conclusão, a densidade de estocagem afeta inversamente o peso corporal e a sobrevivência das larvas nesta espécie.
\end{abstract}

Palavras-chave: peso corporal, densidade, larvas de peixes, Pseudoplatystoma sp, sobrevivência (Fonte: AIMS).

\section{Introducción}

Los bagres rayados (Pseudoplatystoma sp) son especies con alto potencial para acuicultura debido al alto valor económico de su carne para consumo humano y como peces ornamentales; además, presentan altas tasas de crecimiento y su carne es de excelente calidad y de gran demanda nacional e internacional. Sin embargo, por presentar hábitos alimenticios piscívoros desde que eclosionan, presentan altos índices de canibalismo durante su larvicultura y el alevinaje, siendo éste uno de los principales problemas que ha impedido el desarrollo de un paquete tecnológico para su producción a escala comercial (Atencio, 2001; Padilla et al., 2001; Espinal et al., 2005).

La densidad de siembra o alojamiento de los peces en el medio en que se cultivan, influye sobre el comportamiento y altera el apetito, generando competencia agresiva por la comida y espacio, aumentando el canibalismo y facilitando la transmisión de enfermedades
(Hitzfelder et al., 2006 y Treasurer et al., 2011). Por lo anterior, es necesario determinar las características de cultivo más apropiadas para realizar la larvicultura de cada una de las especies que pretendan introducirse a los sistemas de producción. Una de las alternativas para disminuir el canibalismo en larvas de peces, consiste en utilizar sistemas cerrados de recirculación de agua, los cuales han mostrado ser efectivos, tanto para ahorrar agua y espacio, así como para mantener estables las condiciones de calidad del agua requerida para la especie, facilitando el control y la regulación de los factores ambientales. Actualmente estos sistemas son utilizados a escala comercial en cultivos de algunas especies de silúridos como Clarias gariepinus (Akinwoles et al., 2007).

En consecuencia, el presente trabajo evaluó los efectos de la densidad de siembra sobre el peso corporal y la sobrevivencia de larvas de bagre rayado durante el 
período de larvicultura, empleando un sistema cerrado de recirculación operado bajo condiciones controladas de laboratorio.

\section{Materiales y métodos}

Ubicación. El experimento se llevó a cabo en las instalaciones de la Estación Piscícola del Instituto de Acuicultura de la Universidad de los Llanos (IALL-UNILLANOS), ubicada en el kilómetro 12 vía Puerto López, vereda Barcelona, municipio de Villavicencio, departamento del Meta, Colombia; localizado a una altitud de 418 metros sobre el nivel medio del mar, precipitación promedio anual de $4.050 \mathrm{~mm}$ y humedad relativa de $75 \%$.

Sistema de recirculación. Se construyó un sistema cerrado de recirculación con capacidad total de 1400 L, compuesto por 32 contenedores plásticos ovalados con capacidad efectiva de $5 \mathrm{~L}$ cada uno. Cada contenedor fue construido con dos recipientes, colocando uno dentro del otro. El fondo del contenedor interno fue perforado en el fondo con orificios longitudinales $(4 \mathrm{~cm})$, cuyos orificios fueron recubiertos con malla de $300 \mu \mathrm{m}$, suficiente para permitir la salida del agua. Por su parte, el recipiente externo fue perforado en la parte superior para permitir la salida del agua y controlar su nivel en el contenedor interno. El agua, luego de la salida de los contenedores entraba a un filtro de sólidos, construido en malla doble de $200 \mu \mathrm{m}$. Para el tratamiento de los residuos amoniacales se diseñó un biofiltro en cemento con capacidad de $170 \mathrm{~L}$ y caudal $80 \mathrm{~L} / \mathrm{min}$ dividido en seis secciones, las cuales contenían $46 \mathrm{~m}^{2}$ de redes plásticas como sustrato para las bacterias. Para la activación del biofiltro se adicionaron 0.5 gramos de urea/día y se colocaron piedras difusoras durante 20 días, antes del ingreso de las larvas al sistema. Luego del proceso de filtración, el agua era contenida en un tanque de 500L provisto de un detector de nivel, el cual activaba una electrobomba de $3 / 4 \mathrm{HP}$ que elevaba el agua filtrada hasta un tanque de $500 \mathrm{~L}$ desde el cual era nuevamente distribuida hacia los contenedores de larvicultura, los cuales eran abastecidos individualmente con un caudal entre 1.5 a $2.0 \mathrm{~L} /$ minuto. Los contenedores de larvicultura fueron protegidos de la luz solar con una capa de polietileno negro. La aireación la proporcionó un sistema tipo ventury acoplado entre el tanque elevado y el tanque de captación de agua de la electrobomba. Todo el sistema fue soportado en una estructura fabricada con ángulos metálicos con protección anticorrosiva (Figura 1).

Material biológico y alimentación. Se utilizaron 3200 larvas de bagre rayado (Pseudoplatystoma sp), obtenidas por reproducción inducida con Extracto de Hipófisis de Carpa (EHC). Al iniciar la primera alimentación (ca. 50 horas post-eclosión) se trasladaron hasta el Laboratorio de alimento vivo del Instituto de Acuicultura de la Universidad de los Llanos y se sembraron en el sistema cerrado de recirculación a densidades de 10 (T1), 50(T2) y 100(T3) larvas por litro, donde permanecieron hasta los 20 días post-eclosión. El fotoperiodo se mantuvo en oscuridad permanente. Las larvas fueron alimentadas hasta aparente saciedad (Figura 2) con nauplios recién eclosionados de Artemia salina, suministrados en cinco raciones por día (a las 7:00, 9:00, 14:00, 18:00, 22:00 horas). La aparente saciedad fue definida como el momento en el cual aproximadamente el $90 \%$ de las dejaban de perseguir e ingerir los nauplios de Artemia salina suministrados. Para la incubación de los cistos y la cosecha de los nauplios se utilizó el protocolo propuesto por Agh y Sorgeloos (2005). Las larvas fueron pesadas a los 20 días post-eclosión (DPE), empleando una balanza analítica (Ohaus Explorer Pro, sensibilidad. $0.1 \mathrm{mg}$ ), al final del ensayo se registró la sobrevivencia, contando las larvas presentes y calculando su porcentaje con relación al número total de larvas sembradas al inicio del ensayo.

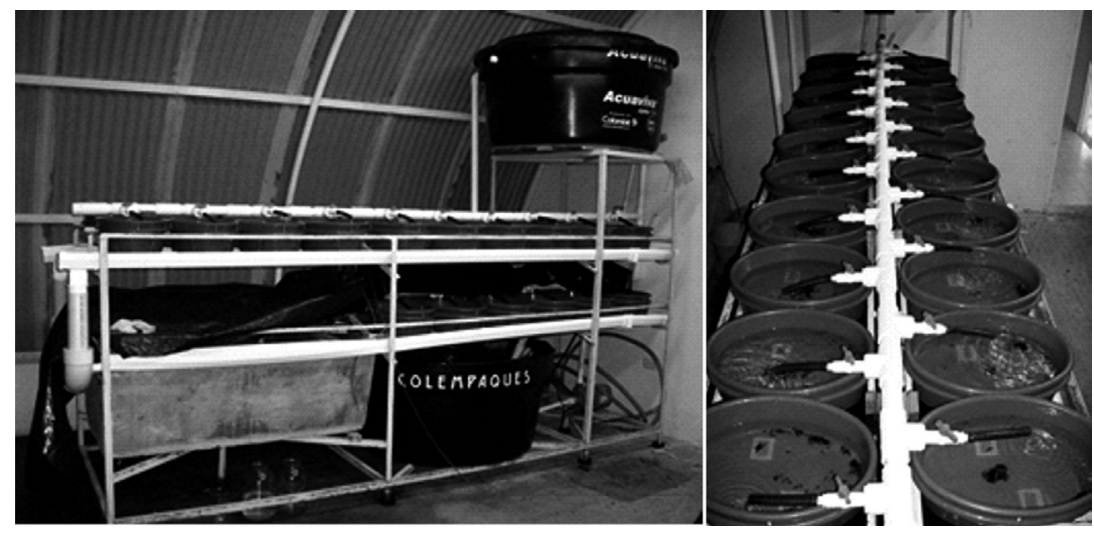

Figura 1. Sistema cerrado de circulación del agua: A. Vista lateral, B. Detalle de los contenedores. 


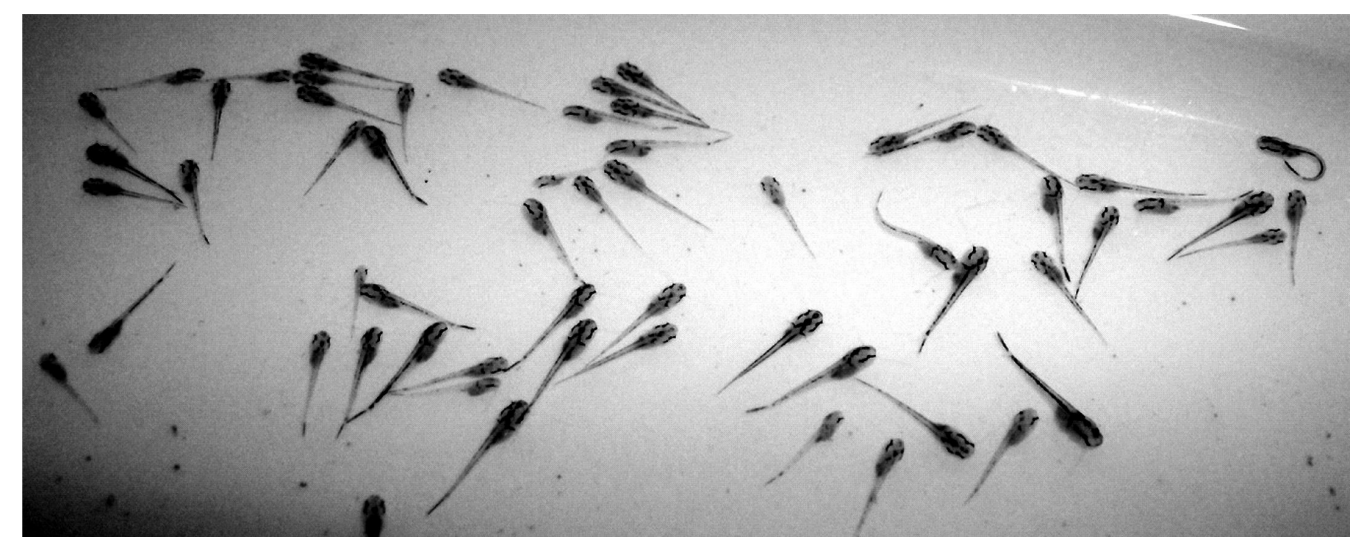

Figura 2. Larvas de bagre rayado 5 días post-eclosión, aparentemente saciadas después de alimentarse con nauplios de Artemia salina.

Monitoreo de calidad del agua. Diariamente, tres veces al día (7:00; 14:00; 20:00 horas) se registraron los siguientes parámetros de calidad de agua: oxígeno disuelto, temperatura, salinidad y conductividad. Para la determinación de estos parámetros se utilizó una sonda multiparamétrica (YSI 556 MPS). Para la determinación de pH se utilizó una sonda YSI pH100. El amonio, dureza y alcalinidad se determinaron a través del kit de análisis de aguas de Aquamerck ${ }^{\circledR}$. Los valores observados fueron: oxígeno disuelto $5.65 \pm 0.26 \mathrm{mg} / \mathrm{L}$; temperatura $26 \pm 0.8^{\circ} \mathrm{C} ; \mathrm{pH} 7.6 \pm 0.2$; dureza $38.24 \pm 5.23$ $\mathrm{mgCaCO} 3 / \mathrm{L}$; alcalinidad $26.47 \pm 5.23 \mathrm{mg} \mathrm{CaCO} / \mathrm{L}$; $\mathrm{NH}_{3}<0.02 \mathrm{mg} / \mathrm{L}$; salinidad 0.42 \pm 0.07 ; conductividad $841.06 \pm 38.12 \mu \mathrm{S} / \mathrm{cm}$; promedios obtenidos de $60 \mathrm{me}-$ diciones), todos estos valores se mantuvieron durante la fase experimental dentro de los rangos de confort para peces tropicales (Yu et al., 2008). Diariamente, a las 19:00 horas, se realizó sifoneo y limpieza de los contenedores para retirar residuos de alimento y larvas muertas. Cada 2 días se cambió el 15\% del agua del sistema.
Diseño experimental y análisis estadístico. Los valores de las variables de calidad de agua fueron expresados como promedio \pm DE. Se utilizó un diseño completamente al azar, con tres tratamientos y cuatro réplicas por tratamiento, para doce unidades asociadas con el modelo experimental y contrastándose el efecto del tratamiento (densidad). Todos los resultados se procesaron en el programa SAS (Statistical Analysis System Institute Inc., versión 9.0), aplicando ANOVA con el 95\% de confianza. Finalmente se empleó la prueba de Tukey para analizar las diferencias entre las medias de los pesos y entre los porcentajes de sobrevivencia. Para determinar diferencias en la sobrevivencia, los datos fueron transformados a arcoseno.

\section{Resultados}

El peso corporal de las larvas fue afectado de manera inversa por la densidad, observándose el mayor peso en aquellas larvas sembradas a la menor densidad
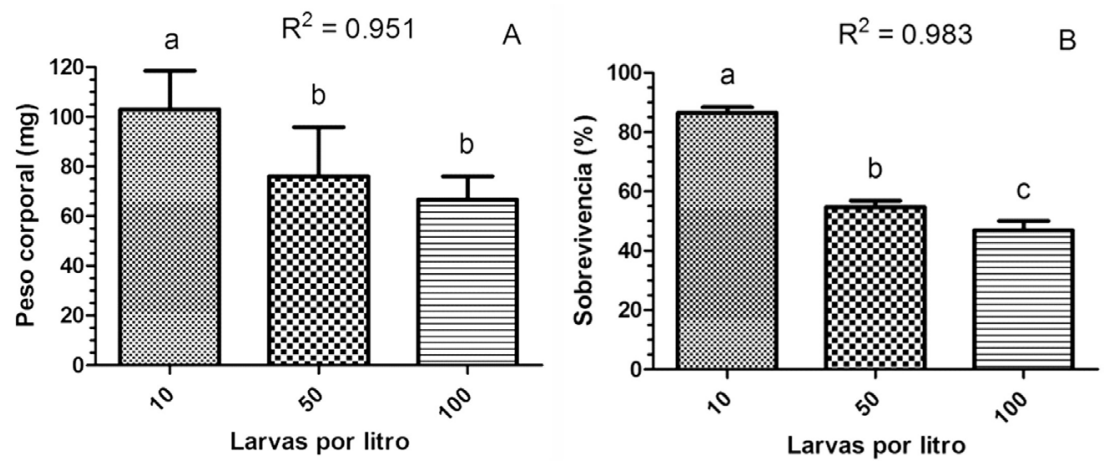

Figura 3. Peso corporal $(A)\left(Y=124.067 e^{-0.218 x}\right)$ y porcentaje de sobrevivencia $(B)\left(Y=85.022 x^{-0.569}\right)$ registrados en larvas de bagre rayado (Pseudoplatystoma sp) cultivadas bajo diferentes densidades de siembra en un sistema cerrado de recirculación. Letras diferentes indican diferencia estadística entre tratamientos $(p<0.01)$. 


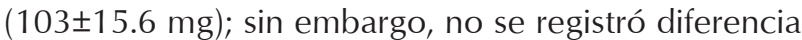
estadística $(\mathrm{P}>0.05)$ entre los tratamientos de 50 y 100 Larvas/L (76.1 \pm 19.8 y $66.7 \pm 9.3 \mathrm{mg}$, respectivamente) (Figura 3A). El mayor coeficiente de variación fue determinado en la densidad intermedia (26.0), mientras que el de la densidad alta fue similar a lo registrado en la densidad baja (14 y 15.2, respectivamente).

El porcentaje de sobrevivencia también fue afectado de manera inversa y significativa $(\mathrm{P}<0.01)$ por la densidad de siembra, registrándose el mayor porcentaje de sobrevivencia con la densidad más baja $(86.5 \pm 1.9 \%)$ seguida por la densidad intermedia $(54.7 \pm 2.2 \%)$ y la más baja sobrevivencia con la mayor densidad (46.9 \pm $3.1 \%$ ) (Figura 3B).

\section{Discusión}

En la mayoría de las especies de peces se ha encontrado una relación inversa entre la densidad de siembra y la tasa de crecimiento, debido a la disminución en la conversión alimenticia producida por el comportamiento jerárquico de dominancia que se establece entre los individuos. Varios factores han sido identificados que afectan la producción de larvas de peces, tales como la calidad del agua, la forma y el volumen de los tanques de cría, la especie cultivada, la etapa fisiológica y el nivel nutricional; sin embargo, el genotipo y el comportamiento social del pez pueden ser considerados como las variables más importantes debido a que son los factores internos que influencian la interacción tasa de crecimiento-densidad (Treasurer et al., 2011). Por esta razón hay especies que tienen un aumento en la tasa de crecimiento cuando se producen en sistemas de policultivo intensivo, como la carpa (Cyprinus carpio) y la tilapia azul (Oreochromis aureus), mientras que existen otras que por el contrario, con un ligero aumento en la densidad de alojamiento, disminuyen drásticamente su crecimiento, inclusive hasta a afectar su sobrevivencia (Papoutsoglou et al., 1992). Por lo tanto, Pseudoplatystoma sp parece ser de éste último tipo de especies de peces, debido a que se observó un efecto negativo del aumento de la densidad de siembre sobre el peso corporal observado al final del experimento $\left(\mathrm{P}<0.01 ; \mathrm{r}^{2}\right.$ 0.951), así como sobre la sobrevivencia $\left(\mathrm{P}<0.01 ; \mathrm{r}^{2} 0.983\right)$; sin embargo, el peso corporal en las densidades más altas (50 y 100 larvas/L) no mostró diferencia estadística significativa. En larvas de la misma especie, a los 12 días post-eclosión se observó un efecto negativo del aumento de la densidad (10, 30 y 100 larvas/L) sobre el crecimiento, pero no sobre la sobrevivencia (Nuñez et al., 2008). Resultados similares fueron observados en larvas de Clarias gariepinus, cuando se contuvieron a densidades entre 25 y 250 larvas/L (Haylor, 1992); sin embargo, Campagnolo y Oliveira (2006) mantuvieron larvas de Pseudoplatystoma corruscans desde 5 hasta 95 larvas/L en un sistema cerrado sin recirculación y encontraron un efecto negativo del aumento de la densidad sobre la sobrevivencia, observando el mejor resultado cuando se utilizaron 15 larvas/L sin registrarse ninguna relación entre la tasa de crecimiento y la densidad. Contradictoramente, en larvas de Heterobranchus longifilis contenidas entre 5 y 50 larvas/L se observó un efecto negativo sobre la ganancia de peso, disminuyéndola y aumentando su desviación estándar, y un efecto positivo sobre la sobrevivencia, presentándose las menores tasas de sobrevivencia en las densidades más bajas (Imorou et al., 2008).

Se concluye entonces, que aunque las condiciones físico-químicas del agua se mantengan controladas, el crecimiento y la sobrevivencia de larvas de bagre rayado son afectados en gran medida por el aumento de la densidad de alojamiento durante la etapa de larvicultura.

\section{Agradecimientos}

A los propietarios y técnicos de las granjas piscícolas Las Brisas y Langostinos del Llano Ltda por permitir el uso de sus instalaciones durante el proceso reproductivo; al profesor José Alfredo Arias Castellanos por los aportes sobre el diseño del sistema de recirculación y al Médico Veterinario Zootecnista Ricardo Murillo Pacheco por su apoyo en la logística.

\section{Referencias}

Agh N, Sorgeloos P. Handbook of protocols and guidelines for culture and enrichment of live food for use in larviculture. Iran: Urmia University; 2005.

Akinwoles AO, Faturoti EO. Biological performance of African Catfish (Clarias gariepinus) cultured in recirculating system in Ibadan. Aquacult Eng. 2007;36:18-23.

Atencio VG. Producción de alevinos de especies nativas. Rev MVZ Córdoba. 2001;6:9-14.

Campagnolo R, Oliveira Nuñer AP. Sobrevivência e crescimento de larvas de surubim, Pseudoplatystoma corruscans (Pisces, Pimelodidae), em diferentes densidades de estocagem. Acta Sci Anim Sci. 2006;28:231-237.

Espinal CF, Martínez HJ, González FA. La cadena de la piscicultura en Colombia, una mirada global de su estructura y dinámica 1991-2005. Ministerio de Agricultura y Desarrollo Rural, Observatorio Agrocadenas Colombia. 2005;72:13.

Haylor GS. Controlled hatchery production of Clarias gariepinus (Burchell): growth and survival of larvae al high stocking density. Aquacult Fish Manage. 1992;23:303-314. 
Hitzfelder GM, Holt GJ, Fox JM, McKee DA. The efffect of rearing density on growth and survival of cobia Rachycentron canadum, larvae in a closed recirculating aquaculture system. J World Aquacult Soc. 2006;37:204-209.

Imorou TI, Fiogbe ED, Kestemont P. Determination of appropriate age and stocking density of vundu larvae, Heterobranchus longifilis (Valenciennes 1840), at the weaning time. Aquacult Res. 2008;39:24-32.

Nuñez J, Dugué R, Arana NC, Duponchelle F, Renno JF, Raynaund T, Hubert N, Legendre M. Induced breeding and larval rearing of Surubí, Pseudoplatystoma fasciatum (Linnaeus, 1766), from the Bolivian Amazon Aquac Res. 2008;39:764-776.
Padilla P, Alcántara B, Ismiño R. Reproducción inducida de la doncella Pseudoplatystoma fasciatum y desarrollo embrionario-larval. Folia Amazon. 2001;12(1-2):141-150.

Papoutsoglou SE, Petropoulos G, Barbieri R, Polyculture rearing of Cyprinus carpio (L.) and Oreochromis aureus (St.) using a closed circulated system. Aquaculture. 1992;103:311-320.

Treasurer J, Atack T, Rolton A, Walton J, Bickerdike R. Social, stocking density and dietary effects on the failure of farmed cod Gadus morhua. Aquaculture. 2011;322-323:241-248.

Yu C, Xing B, Xu L, Li D. Water quality management in intensive aquaculture in china. En: IFIP International Federation for Information Processing, Volume 259; Computer and Computing Technologies in Agriculture, Vol 2; Daoliang Li, (Boston: Springer); 2008;143-1252. 